hep-th/9704123

UTTG-14-97

SLAC-PUB-7462

April 1997

\title{
M(atrix) String Theory on K3
}

\author{
Willy Fischler $\diamond$ 用, Arvind Rajaraman`四 \\ $\diamond$ Theory Group,Department of Physics, University of Texas, Austin, TX 78712. \\ - Stanford Linear Accelerator Center, Stanford, CA 94309
}

\begin{abstract}
We conjecture that M-theory compactified on an ALE space (or K3) is described by 0-branes moving on the ALE space. We give evidence for this by showing that if we compactify another circle, we recover string theory on the ALE space. This guarantees that in the large $\mathrm{N}$ limit, the matrix model correctly describes the force law between gravitons moving in an ALE background. We also show the appearance in $M($ atrix) theory of the duality of M-theory on K3 with the heterotic string on a three-torus.
\end{abstract}

Submitted to Nuclear Physics B.

\footnotetext{
*e-mail address: fischler@utaphy.ph.utexas.edu

$\dagger^{\dagger}$ e-mail address: arvindra@dormouse.stanford.edu

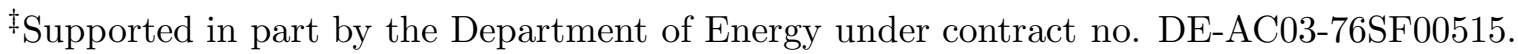




\section{INTRODUCTION}

M(atrix) theory [1] has been proposed as a nonperturbative formulation of M-theory. The conjecture is that M-theory in the infinite momentum frame is described by a Hamiltonian that consists of zero-branes and their interactions. These zero-branes are the carriers of longitudinal momentum. We shall refer to these partons as $\tilde{D} 0$-branes . This conjecture has passed many tests. The Hamiltonian contains 11-dimensional supergravitons and supermembranes in its spectrum and reproduces their interactions [1 1 . It also possesses T-duality upon compactification [5],6]. In addition, upon compactifying on a circle and shrinking the circle to zero radius, $\mathrm{M}($ atrix) theory has been shown [7-10 to contain multistring states. These strings interact according to the usual light-cone interactions and the proper scaling between the coupling constant and the radius is recovered 9 .

It is important to consider compactifying the $\mathrm{M}$ (atrix) model on more complicated surfaces which preserve less supersymmetry. The natural first step is to consider the simplest Calabi-Yau space, the four-dimensional $K 3$ surface. It is a reasonable guess that M-theory on $K 3$ in the infinite momentum frame is described by the dynamics of $\tilde{D} 0$-branes moving on $K 3$ [11].

However, in [12], the authors computed the force law between two $\tilde{D}$ 0-branes moving on $K 3$. They found that the $v^{4}$ part of the force was not the same as the force expected from supergraviton exchange. This appeared to signal a potential problem with this model.

We believe that this disagreement is not a fatal one. There is no nonrenormalization theorem for the gravitational coupling constant in $\mathcal{N}=2$ theories. Therefore the coupling constant could easily be different at short and large distance scales. In any case, for the connection to M(atrix) theory, we need to consider the interaction of bound states of large numbers of $\tilde{D} 0$-branes since it is only in the large $N$ limit that one recovers the force law derived from supergravity.

In order to show how the large $N$ limit is involved in this context and to simplify the discusssion, we will begin by following the proposal of [11] for a matrix model of M-theory 
on an ALE surface. We will then compactify an additional circle. In the limit where this circle is small, (keeping the ALE size fixed in string units), we will show how to recover type IIA strings propagating in an ALE background. String theory then guarantees that the gravitational force law is exactly reproduced in M(atrix) theory without the need for

additional degrees of freedom beyond the $\tilde{D} 0$-branes. Since the force law between gravitons is reproduced in light-cone string theory only when $N \rightarrow \infty$, there is no conflict with the results of [12].

Finally, we subject this model to another test. In the limit when the volume of the $K 3$ shrinks to zero, string duality predicts that we should get the dynamics of heterotic strings on $T^{3}$. We show that this is indeed the case, thus providing another consistency check of this model.

\section{M(ATRIX) STRING THEORY}

We will review the calculation of [9], expressing it in terms that will be suitable for us. The bosonic part of the M(atrix) theory hamiltonian is

$$
H=R_{11} \operatorname{tr}\left(\Pi_{i}^{2}+\left[X^{i}, X^{j}\right]^{2}\right)
$$

On compactification on a circle of radius $R_{9}$, the $\mathrm{M}$ (atrix) model is described by a $1+1$ dimensional gauge theory [13] with the Hamiltonian

$$
H=\int^{2 \pi} \frac{d \sigma}{R_{9}} \operatorname{tr}\left(\Pi_{i}^{2}+R_{9}^{2}\left(D X^{i}\right)^{2}+\frac{1}{R_{9}^{2}} E^{2}+\left[X^{i}, X^{j}\right]^{2}\right)
$$

Rescaling $X^{i} \rightarrow R_{9}^{-1 / 2} X^{i}$, we get

$$
H=\int^{2 \pi} d \sigma \operatorname{tr}\left(\Pi_{i}^{2}+\left(D X^{i}\right)^{2}+\frac{1}{R_{9}^{3}}\left(E^{2}+\left[X^{i}, X^{j}\right]^{2}\right)\right)
$$

For small $R_{9}$, the type IIA limit, we find that the terms $[X, X]$ and $E$ must be set equal to zero. The condition $[X, X]=0$ implies that we are on the moduli space of the theory where the $X^{i}$ commute. The condition $E=0$ implies that the gauge dynamics is such that 
only gauge-singlet states survive. The objects which are gauge-invariant are the eigenvalues of the matrices $X^{i}$. Accordingly, the small $R_{9}$ dynamics is reduced to the dynamics of the eigenvalues of $X^{i}$. This can be described as a theory with $Z_{N}$ gauge invariance.

The $X^{i}$ are functions of the coordinate $\sigma$ in the YM theory. Due to the residual $Z_{N}$ symmetry, they do not have to be periodically identified, but rather only identified upto a permutation of the eigenvalues. This effectively multiplies the length of the string by $N$. We can make strings of any longitudinal momentum by this procedure.

The world sheet Hamiltonian reduces to

$$
H=\int^{2 \pi} d \sigma \operatorname{tr}\left(\Pi_{i}^{2}+\left(\partial X^{i}\right)^{2}\right)
$$

Thus the long strings which are produced have the dynamics of $I I A$ strings.

We now turn to the problem we are interested in, which is the M(atrix) model compactified on $K 3$. We shall start with a simpler case, where we have an ALE surface instead of K3. The conjecture is then that M-theory on an ALE surface is described by the dynamics of 0-branes on the ALE. We will start by working in the orbifold limit of the ALE surface. Coordinates will be chosen so that $\left(x_{6}, x_{7}, x_{8}, x_{9}\right)$ are the directions along the ALE surface.

The dynamics of 0-branes on an ALE space was studied in great detail in [15], 16]. For illustrative purposes, we will take the example $R^{4} / Z_{2}$. In this case, each 0-brane has an image. We therefore take $N$ 0-branes and their images under $Z_{2}$ moving on $R^{4}$. One then quantizes the open strings connecting these 0-branes and then imposes invariance under $Z_{2}$.

There are two types of strings connecting these branes, those with polarizations along the ALE surface (which we will call $\psi^{a}, a=6,7,8,9$ ) and those with polarizations perpendicular to the ALE surface (which we call $X^{i}, i=0,1,2,3,4,5$ ). Each of them is represented by a matrix in $U(2 N)$. One then imposes the $Z_{2}$ projection on these states.

We can schematically write the $Z_{2}$ projection as

$$
U X^{i} U^{-1}=X^{i} \quad U \psi^{a} U^{-1}=-\psi^{a}
$$

where $U$ is a unitary operator representing the $Z_{2}$. 
We can represent the solution of these equations by

$$
X^{i}=\left(\begin{array}{cc}
A^{i} & B^{i} \\
B^{i} & A^{i}
\end{array}\right) \quad \psi^{a}=\left(\begin{array}{cc}
C^{a} & D^{a} \\
-D^{a} & -C^{a}
\end{array}\right)
$$

where $A^{i}, B^{i}, C^{a}, D^{a}$ are $N \times N$ matrices.

In order to derive the Hamiltonian describing the dynamics of zero-branes, it is useful to start with a 6 -dimensional theory since the zero-branes move on $R^{6} \times$ ALE. The resulting theory, thought of as a $\mathcal{N}=1, d=6$ theory, has $U(N) \times U(N)$ gauge symmetry, and there are (in addition to the vector multiplet), two hypermultiplets transforming in the $(N, \bar{N})$ representation. The theory on the world-volume of the zero-branes is then the dimensional reduction of this gauge theory to $0+1$ dimensions.

We are interested in the case where in addition, we compactify on a circle of radius $R_{9}$. We can T-dualize along the circle in order to get a theory of 1-branes wrapped on the dual circle. After doing this the Hamiltonian is [15, 16]

$$
\begin{array}{r}
H=\int \frac{d \sigma}{R_{9}} \operatorname{tr}\left(R_{9}^{-2} E^{2}+P_{X}^{2}+P_{\psi}^{2}+R_{9}^{2}\left(D X^{i}\right)^{2}+R_{9}^{2}\left(D \psi^{a}\right)^{2}+\right. \\
\left.\sum_{a, b}\left|\left[\psi^{a}, \psi^{b}\right]\right|^{2}+2 \sum_{a, i}\left|\left[\psi^{a}, X^{i}\right]\right|^{2}+\sum_{i, j}\left|\left[X^{i}, X^{j}\right]\right|^{2}\right)
\end{array}
$$

where commutators are taken in the $U(2 N)$ matrices.

We rescale $X^{i} \rightarrow R_{9}^{-1 / 2} X^{i}, \psi^{a} \rightarrow R_{9}^{-1 / 2} \psi^{a}$.The Hamiltonian is then

$$
\begin{gathered}
H=\int d \sigma \operatorname{tr}\left(P_{X}^{2}+P_{\psi}^{2}+\left(D X^{i}\right)^{2}+\left(D \psi^{a}\right)^{2}+\frac{1}{R_{9}^{3}} E^{2}\right. \\
\left.\frac{1}{R_{9}^{3}}\left[\sum_{a, b} \operatorname{Tr}\left|\left[\psi^{a}, \psi^{b}\right]\right|^{2}+2 \sum_{a, i} \operatorname{Tr}\left|\left[\psi^{a}, X^{i}\right]\right|^{2}+\sum_{i, j} \operatorname{Tr}\left|\left[X^{i}, X^{j}\right]\right|^{2}\right]\right)
\end{gathered}
$$

In the limit $R_{9} \rightarrow 0$, to analyse the light spectrum, we must set $E=0$ and also set the potential terms to zero. The vanishing of $E$ again implies that the effective theory will be described in terms of gauge-singlet objects .

The vanishing of the potential terms implies that we are on the moduli space. In this case, the moduli space is more complicated; we have both a Higgs and a Coulomb branch. We will analyse them separately. 
The Higgs branch is the branch where $\psi \neq 0$. Since $\psi$ denotes the motion within the ALE space, this implies that the D-branes have moved off the fixed point. The $Z_{2}$ projection implies that the images must then be at the same point in the transverse space i.e. the D-brane and its image are at positions $\left(x_{2}, x_{3}, x_{4}, x_{5}, x_{6}, x_{7}, x_{8}, x_{9}\right)$ and $\left(x_{2}, x_{3}, x_{4}, x_{5},-x_{6},-x_{7},-x_{8},-x_{9}\right)$ respectively.

In general, different pairs of D-branes do not have to be at the same value of $\left(x_{2}, x_{3}, x_{4}, x_{5}\right)$. We can therefore separate the $2 N$ D-branes into $N$ pairs.

For each pair of D-branes, we need to find a set of gauge-invariant coordinates parametrizing their position on the Higgs branch. We must first impose the condition that the potential energy vanishes. We must then choose a gauge invariant set of coordinates.

It was shown in [15, 16] that after these operations, we have precisely four gauge-invariant scalars $Y^{a}$ left. One can then find the dynamics for these scalars. It was shown in 15, 16 that these scalars propagate according to the Lagrangian $g_{a b} \partial Y^{a} \partial Y^{b}$, where $g_{a b}$ coincides with the ALE metric: $g_{a b}=g_{a b}^{A L E}$.

This is true for a general ALE space, i.e. after imposing the vanishing of the potential terms and gauge invariance, we have four gauge-invariant coordinates which have a nontrivial moduli space metric, which is identical to the ALE metric.

We now turn to the transverse coordinates. Each pair of D-branes was at a transverse coordinate $\left(x_{2}, x_{3}, x_{4}, x_{5}\right)$. If we take $N$ pairs of 0 -branes, we will have a $N \times N$ matrix parametrizing these positions. The gauge invariant objects are the eigenvalues of these matrices.

The dynamics of these eigenvalues will determine the propagation of the string in the transverse coordinates. Accordingly, one expects a trivial metric in the kinetic terms. We have not been able to prove this directly, but this seems very plausible for several reasons. The eigenvalue dynamics is a $1+1$ sigma model. It is well known that such a theory flows to a Ricci flat metric in the IR. Furthermore, at the classical level, the metric is trivial. There are no one-loop corrections [12]. Supersymmetry then implies that the flat metric is not corrected perturbatively. It seems reasonable to suppose that the non-perturbative 
corrections will still allow the sigma model to flow to a free theory in the IR. We shall therefore make this assumption.

As before, we have a $Z_{N}$ gauge symmetry. We can therefore produce long strings by identifying the matrices upto a permutation of eigenvalues when we go around the YM circle. These strings will have a world-sheet Lagrangian

$$
L=\left(\partial X^{i}\right)^{2}+g_{a b}^{A L E} \partial Y^{a} \partial Y^{b}+\text { fermions }
$$

which is precisely that of a string propagating in an ALE background.

We turn now to the Coulomb branch. On this branch, the $\psi^{a}$ are set equal to zero, which means that the 1-branes are stuck to the fixed point. The $X^{i}$ can have arbitrary values. These states were shown [15,24,11] to correspond to the extra gauge bosons needed to enhance the gauge symmetry to a nonabelian group.

Hence we have obtained all the states of type IIA string theory in the background of an ALE space. The consistency of string theory will then automatically provide the force law between two gravitons. Although this is a consistent theory for finite $N$ [23], the full Lorentz invariance is only recovered in the large $N$ limit. One should therefore expect to recover the force law between two gravitons only in this limit..

One must also show that the structure of the vertex is of the same form as in string theory. To do this requires a more detailed analysis of the superconformal field theory. We expect that supersymmetry is sufficient to recover the vertex structure, but a demonstration of this is desirable.

So far we have only looked at the orbifold limit. The case where the orbifold singularity is blown up was also considered in [15, 16], where it was shown that the blowup corresponded to the addition of Fayet-Iliopoulous D-terms. Remarkably, the result is very similar; the metric on the Higgs branch is altered so that it is still identical to the metric on the nonsingular ALE space! Thus everything we have said goes through unchanged. We still recover string theory in an ALE background, and the force law is guaranteed to work in the large $N$ limit. When we have the full $K 3$ instead of an ALE space, there are certain complications that 
arise. The compactification of M-theory on $K 3$ seems to be described by a $4+1$ dimensional field theory, like the case of compactification on $T^{4}$ which is a $4+1 \mathrm{YM}$ theory. These theories are difficult to define.

Presently, we can only discuss M-theory near the orbifold points of $K 3$ where the dynamics is simple enough to enable us to make statements. At these points, the $K 3$ is flat everywhere except near the orbifold singularities, where the geometry is that of the ALE space. We have already seen that 0-branes moving in an ALE space see the ALE space geometry. It is obvious that 0-branes in flat space see flat space geometry. Putting these facts together, we see that, at least near the orbifold limit, the D-branes see the geometry of $K 3$.

We can then go through the procedure of constucting strings on $K 3$ in exact parallel to the discussion for the ALE space.The only difference is that on the Higgs branch, the moduli space metric is the $K 3$ metric instead of the ALE metric. Thus the strings we construct will have exactly the worldsheet action of strings in the background of $K 3$. This again shows that the force law between two gravitons will work out correctly in the large $N$ limit.

\section{M-THEORY-HETEROTIC DUALITY}

In the limit where the volume of the $K 3$ shrinks to zero, we expect to recover heterotic string theory on $T^{3}$ [17]. We now show how this occurs in M(atrix) theory.

The idea is very simple. We are looking at the dynamics of $\tilde{D} 0$-branes in the background of a shrinking $K 3$. It is natural to T-dualize this theory so that it becomes a theory of 4branes wrapped on $K 3$. It turns out that these 4-branes are at strong coupling, so it is natural to interpret this as a theory of M-theory 5-branes wrapped on $K 3$. The extended dimension of the 5 -branes is much longer than the $K 3$ dimensions. We can therefore treat it as effectively being a $1+1$ dimensional theory, with a Lagrangian following from wrapping a 5-brane on $K 3$. This is known to have the same world-sheet structure as a heterotic

string [18,[19]. We will therefore recover the heterotic string theory in this limit, exactly as 
expected.

We would like to see that the tension of the heterotic string obtained in this manner is exactly the value expected from M-theory-heterotic duality.

To simplify the calculation, we first consider toroidal compactification along the lines of [20], where M-theory is compactified on a four-torus with sides $L_{i}, i=1,2,3,4$. This is represented in $\mathrm{M}$ (atrix) theory by a $4+1$ dimensional Yang-Mills theory on a four torus of radii $\Sigma_{i}=(2 \pi)^{2} \frac{l_{11}^{3}}{R L_{i}}, i=1,2,3,4$, with a coupling constant $g^{2}=(2 \pi)^{6} \frac{l_{11}^{6}}{R L_{1} L_{2} L_{3} L_{4}}$.

It was shown in [14 that the coupling constant becomes an extra dimension. Thus we reach a $5+1$ dimensional theory. This is interpreted as the theory on the world-volume of $N$ M-theory 5 -branes wrapped on a 5 -torus with sides $\Sigma_{i}=(2 \pi)^{2} \frac{l_{11}^{3}}{R L_{i}}, i=1,2,3,4$, and $\Sigma_{5}=(2 \pi)^{5} \frac{l_{11}^{6}}{R L_{1} L_{2} L_{3} L_{4}}$.

This $5+1$ dimensional theory is still somewhat mysterious, but we can still extract some information. In particular, if we shrink four out of the five dimensions to zero size, we reach a $1+1$ dimensional theory describing a string. We can do this in five ways, thus obtaining five strings. Four of these correspond to membranes wrapped on the four circles of the $T^{4}$. The fifth is the 5 -brane wrapped on $T^{4}$. There is a symmetry among these five strings. This is related to the symmetry used in [21,22] to give a construction of the five-brane wrapped on $T^{5}$.

We already know the tensions of the four strings corresponding to the four membranes. The string tensions corresponding to these strings are given by $T_{s}=\frac{1}{R \Sigma_{i}}, i=1,2,3,4$. The symmetry then tells us that the fifth string will have tension $T_{s}=\frac{1}{R \Sigma_{5}}=\frac{L_{1} L_{2} L_{3} L_{4}}{(2 \pi)^{5} l_{11}^{6}}$, which is the right tension for a five-brane wrapped on $T^{4}$.

We can perform exactly the same manipulations for M-theory on a $K 3$ of volume $v l_{11}^{4}$. This, according to our conjecture, is a theory of $\tilde{D} 0$-branes moving on this $K 3$. After Tduality [25], this is described by a $4+1$ dimensional theory on a $K 3$ of volume $\frac{l_{11}^{12}}{R^{4}\left(v l_{11}^{4}\right)}=\frac{l_{11}^{8}}{R^{4} v}$. This is equivalent to a $5+1$ dimensional theory, which we interpret as the world-volume of $N$ M-theory 5 -branes wrapped on $K 3 \times S^{1}$. The length of the $S^{1}$ is, as before, $\Sigma_{5}=(2 \pi)^{5} \frac{l_{11}^{2}}{R v}$. In the limit $v \rightarrow 0$, this is a $1+1$ dimensional theory where the 5 -branes are wrapped on a 
small $K 3$ and a large $S^{1}$.

Although we do not know the world-volume theory of the 5-brane, it is very reasonable to assume that the dynamics will force us onto the moduli space of the theory. This means that the $N$ 5-branes will be separated from each other in the transverse space. Each 5-brane is wrapped on a very small $K 3$, leading to a $1+1$ dimensional theory which is known to be a theory which describes the worldsheet action of the heterotic string [18,19]. As usual we have a $Z_{N}$ symmetry, and we can go through the usual procedure of making long strings.

The string scale of the heterotic string is, as before, $T_{s}=\frac{1}{R \Sigma_{5}}=\frac{v}{(2 \pi)^{5} l_{11}^{2}}$, which is the correct value predicted from string duality.

It would be interesting to see the connection of this construction to the matrix model proposed for heterotic strings [10].

\section{ACKNOWLEDGEMENTS}

We are grateful to E.Halyo and especially to L.Susskind for crucial discussions.

W. F. thanks the ITP at Stanford for its hospitality and was supported in part by the Robert Welch Foundation and NSF Grant PHY-9511632. A.R. was supported in part by the Department of Energy under contract no. DE-AC03-76SF00515. 


\section{REFERENCES}

[1] T. Banks,W. Fischler,S. Shenker and L. Susskind,hep-th/9610043.

[2] M.R.Douglas, D.Kabat, P.Pouliot and S.Shenker, hep-th/9608024.

[3] G. Lifschytz, hep-th/9612223.

O. Aharony and M. Berkooz, hep-th/9611215.

G. Lifschytz and S. Mathur, hep-th/9612087.

[4] J. Polchinski and P. Pouliot, hep-th/9704029.

[5] L. Susskind,hep-th/9611164.

[6] O.J. Ganor, S. Ramgoolam and W.Taylor,hep-th/9611202.

[7] L. Motl,hep-th/9701025.

[8] T. Banks and N. Seiberg,hep-th/9702187.

[9] R. Dijkgraaf, E. Verlinde and H. Verlinde, hep-th/9703030.

[10] S.Kachru and E.Silverstein, hep-th/9612162.

T. Banks and L.Motl, hep-th/9703218.

D.Lowe, hep-th/9704041.

N.Kim and S.J. Rey, hep-th/9701139.

[11] M.R. Douglas, hep-th/9612126.

[12] M.R. Douglas, H. Ooguri and S. Shenker, hep-th/9702203.

[13] W. Taylor,hep-th/9611042.

[14] M. Rozali,hep-th/9702136.

[15] M.R. Douglas and G. Moore, hep-th/9603167.

[16] C.V. Johnson and R. Myers, hep-th/9610140. 
[17] E. Witten, Nuc. Phys. B443 (1995) 85.

[18] J. Harvey and A. Strominger, Nuc.Phys. B449 (1995) 535, hep-th/9504047.

[19] S. Cherkis and J. Schwarz, hep-th/9703062.

[20] W. Fischler, E. Halyo, A. Rajaraman and L. Susskind, hep-th/9703102.

[21] E. Halyo, hep-th/9704086.

[22] M. Berkooz and M. Rozali, hep-th/9704089.

[23] L. Susskind, hep-th/9704080.

[24] J. Polchinski, hep-th/9606165.

[25] K. Hori and Y.Oz, hep-th/9702173. 